\title{
EMPLOYING BIM TOOLS TO STREAMLINE FABRICATION
}

\author{
Samuel Goodwin ${ }^{1}$, and Steven K. Ayer ${ }^{2}$
}

\begin{abstract}
Previous research suggests that the building industry has lagged behind manufacturing industries in productivity. This research addresses this challenge by developing a methodology to facilitate prefabrication related to surface penetrations using commercially available Building Information Modeling tools. The simple proof of concept presented in this work illustrates that this approach can provide useful and reliable fabrication information that can be applied to several trades or machine types. In this study, Revit, Dynamo, and other office tools were used to extract and manipulate the desired data. The applications were built, tested, and verified for countertop surface penetrations because countertops rarely have the exact same penetration layout for different projects. Therefore, there is typically a need to manually calculate locations for penetrations. The proof of concept developed in this work was able to instantly determine critical design parameters and calculate relevant penetration locations for fabricators. The accuracy of the outputted values was manually verified. While this particular application might only save a few minutes of a fabricator's time because of the simple design example explored, there could be potential to save more time on more complex designs, which would take longer to manually calculate.
\end{abstract}

Keywords: Prefabrication, BIM, Dynamo, Revit.

\section{INTRODUCTION}

In keeping with the lean construction principles, a production system must be designed for flow and value, as defined by the end user of the process outcome (Goodman, 2009). In this study, the targeted values consist of the following: reducing process variability, reducing cycle time, reducing batch sizes, increasing system flexibility and adding standardization while continuously looking for process improvements (Yoders, 2014). This research explores how component fabrication can be streamlined to advance construction productivity even when the same design is not being mass-produced. Therefore, this research addresses the following questions:

- Can commercially available Building Information Modeling (BIM) software be modified to instantly produce updated and useable information for the purposes of component prefabrication?

- What is the process for modifying such software to produce the desired information?

1 Graduate Research Assistant, School of Sustainable Engineering and the Built Environment, Arizona State University, Tempe, Arizona, USA. sdgoodw1@asu.edu

2 Assistant Professor, School of Sustainable Engineering and the Built Environment, Arizona State University, Tempe, Arizona, USA. sayer@asu.edu 
To address these questions, this research develops a methodology to automatically generate the data necessary to assist in fabricating surface penetrations within a building element. As a proof-of-concept, countertop openings such as sinks, faucets, and waste receptacles are explored.

\section{BACKGROUND}

While productivity in the manufacturing industry has more than doubled in the last 40 years, the productivity of the construction industry has not generally improved (Teicholz, 2003). This has led companies to leverage technology to increase productivity and adopt digital modeling and computer controlled fabrication, which have been used in the manufacturing industry for decades (Allphin, 2014). Construction companies are now using BIM to facilitate and streamline component fabrication for elements such as structural steel and electrical systems (Teicholz, 2014, and Henderson, 2015).

While some BIM-based design authoring tools are starting to become common in different regions in the world, the information needed from those tools often varies for different prefabricators. In this work, Autodesk Dynamo was used in conjunction with Revit to aim to customize information for the needs of a particular prefabricator. Revit, like most other BIM authoring tools, allows designers to create digital building models that contain both geometric and performance information. Dynamo enables visual programing to interact with designs in Revit. While Dynamo is often used for design, this work aims to use it to extract and manipulate information embedded in the Revit model to aid prefabrication. With the right process implementation methodology, BIM and digital fabrication have the potential to improve the building industry through automation (Williams, 2008).

\section{Methodology}

The approach of the proposed methodology is to streamline prefabrication tasks by leveraging existing BIM tools. This particular work explores this topic through the lens of surface penetration fabrication, specifically countertop penetrations. From discussions with countertop fabricators in the Southwest United States, it was observed that their prefabrication process requires a substantial amount of human input. Part of this input requires mathematical calculations that could be automated in order to allow the humans to spend more time analyzing decisions that are difficult to automate (Ernstrom, 2006).

To bridge the gap between constructability and manufacturability of a design, the proposed methodology tests the applicability of BIM to help in fabricating or prefabricating surface penetrations for openings in countertops. To streamline the automation of this process, Revit is used to design the countertop and Dynamo is used to extract useful information from the model in order to produce the information needed for the fabrication of the penetrations needed for the designed openings. This methodology has three high level phases consisting of: preliminary analysis; defining fabrication variables; and implementation and validation of BIM tools. Each phase builds on the thorough and successful execution of the one prior.

\subsection{Preliminary Analysis}

The first analysis step required the researchers to understand how components are currently built. To do this, the researchers observed the current practices for fabricating countertops. The process started with obtaining design drawings. The project engineer 
then used a software package called "Cabinet Vision" to redraw every assembly while referencing casework elevations. The next step was to submit these shop drawings and wait for design approval. Once approved, the engineer used the shop drawings in the same software package to produce fabrication drawings. The software also had a built-in material optimization feature, which maximized the use of each piece of raw material.

In the case of countertop fabrication, every time a penetration was required to create a surface opening, the engineer had to manually determine the opening location and input its dimensions. This may only seem like a minor inconvenience at first, but has the potential to be more critical when considering: the frequency at which this takes place; the time it consumes; and the possibility of human error during the process. The project and the operation are best served by automating this process to enable the engineer to focus on tasks that a computer is incapable of doing.

\subsection{Defining Fabrication Values}

After identifying the tasks that required manual calculation of critical penetration location measurements, the specific values needed for this calculation were determined. In a countertop design, there are many different types of penetrations that may exist such as: sinks, soap dispenser holes, faucet holes, or holes for cooktops, to name a few. While these different penetrations serve different purposes and have different sizing requirements, the process of determining the location of these penetrations is similar. Therefore for this work, sinks were explored because of the commonality of sink penetrations and also because of the various different types and locations of sinks that could create a challenge for repeated manual calculation.

In the case of this work, four different dimensions were inputted to the $\mathrm{CNC}$ software. Two of the dimensions (width and depth) did not require calculations as they were listed on the original design documentation. There were two other dimensions that did require manual calculation. These dimensions are shown in Figure 3.1 as the values of " $X$ " and "Y" variables. The "X" dimension is the distance from the center of the opening to the top left corner forming the hypotenuse of a right triangle. The "Y" dimension is the distance from the center of the opening straight to the back side of the countertop, forming a right leg of the right triangle.

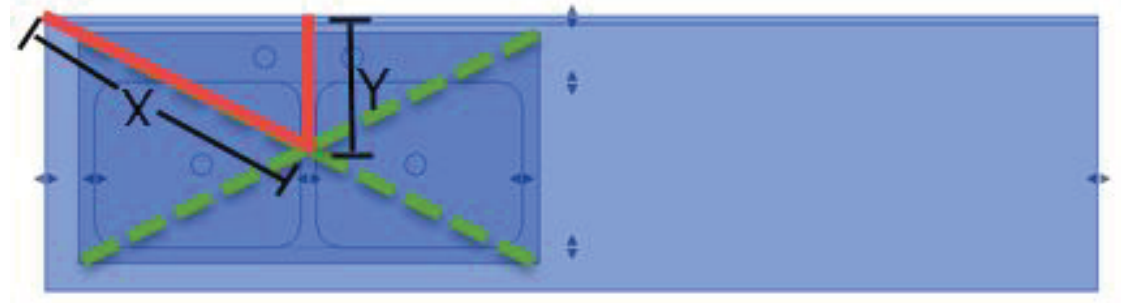

Figure 3.1 Shows the $\mathrm{X}$ and $\mathrm{Y}$ dimensions which have to be calculated manually.

\subsection{Implementation of BIM Tools}

After identifying the parameters that required manual calculation, this step aimed to leverage existing BIM tools to automatically calculate values to eliminate the need for repeated manual calculation. To simulate this workflow, a sample countertop was designed in Revit. This countertop had a sink and corresponding sink penetration modeled. As the sink location and size would shift in the design, the relevant penetration position parameters would change. Dynamo was used to extract the related parameters related to the sink penetration. The exact dimensions that were sought according to the 
prior step, which identified critical location parameters, were not automatically generated by Revit. Therefore, Dynamo was set up to first extract the parameters, which were defined in Revit, and then calculated the unknowns based on basic geometric equations. This automated process effectively recreating the process that had traditionally been performed through manual calculation. When the desired parameters were calculated through Dynamo, they could either be displayed in the Dynamo window or exported to Microsoft Excel in a Comma Separated Value (CSV) format. In striving to simulate a real-world context, the proof of concept developed exported this fabrication information to Excel to demonstrate that this approach could potentially be used even in situations where fabricators may not have direct access to Revit.

To validate this implementation, the values generated by the proof of concept example were manually verified. Furthermore, the countertop design was changed in several different ways to see if the values would indeed be updated. Each time the design was modified, the values generated were also verified by manual calculation. Each verification activity checked for accuracy and reliability.

\section{RESUlts}

The process for generating the fabrication information through the modification and implementation of the BIM tools was achieved through the use of 3 existing software tools. Design information was extracted from Revit through the use of Dynamo. Dynamo was used to manipulate the relevant design parameters so that they would be useful to countertop fabricators, based on their determined needs. Finally, the information generated by Dynamo was exported to Excel to allow it to be accessed by fabricators who may not have access to Revit.

The countertop model (shown in Figure 3.1) was developed in Revit. The input parameters in this example included: the countertop dimensions; and the sink opening dimensions. Location parameters about the opening are also provided by the Revit model, but these parameters required mathematical manipulation in order to be immediately useful for fabrication.

The parameters provided by Revit were mathematically manipulated in Dynamo, as shown in Figure 4.1. For this simple proof-of-concept, only basic arithmetic and the Pythagorean Theorem equations were required to generate useful numbers for fabrication. The nodes in the Dynamo graph were commanded to extract parameter values by name from a model element. The parameter values extracted included: Sink Location; Sink Location to Wall; Sink Opening Depth; and Sink Opening Width. After developing the appropriate algorithm with these parameters in Dynamo, the results were verified by hand computations and proved to be accurate. To further validate the developed algorithm, the developed countertop design was altered several times in various different ways to illustrate alternate sink penetration locations. After each time, the process outcome was verified by manual calculations and the results were found to be accurate and reliable. Each manual calculation took approximately three minutes as opposed to an instant of a mouse click. 


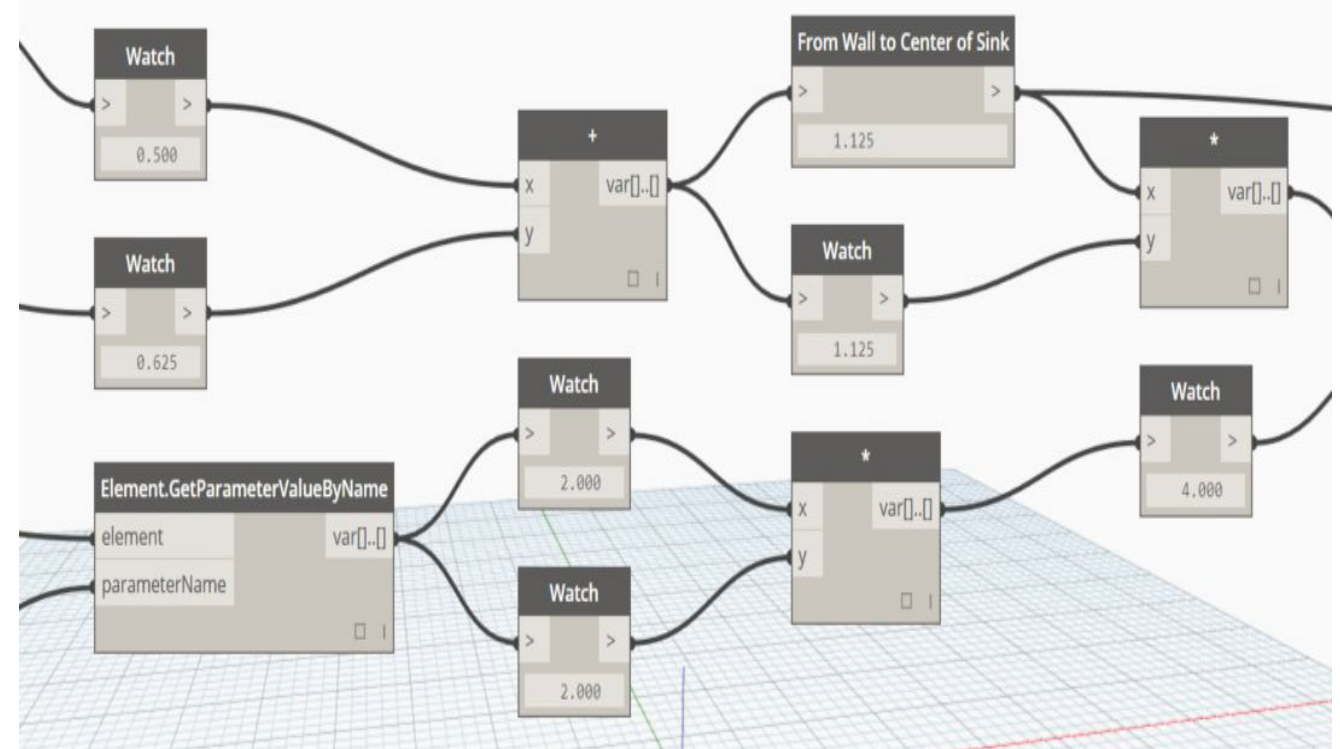

Fig. 4.1: Dynamo was used to extract relevant parameters from Revit design;

Mathematically manipulate those parameters to offer value for prefabrication; and Export the outputted values to a spread sheet.

Once the manipulation tasks were performed in Dynamo, and the algorithm was validated, the results were exported to an Excel file to provide all the fabrication input values that were traditionally calculated manually. This process was checked to ensure that it worked effectively and accurately. To perform this validation, the countertop design was modified several times and the resulting Excel file was checked to ensure that the numbers produced matched those calculated by hand.

\section{DISCUSSION}

The developed approach for using existing BIM-based software tools to aid prefabrication efforts was shown to be possible in a countertop example. It is likely that, in this particular example, this basic application could save time through repeated use. However, there is a limitation to this work because it only explored a simple, proof-ofconcept application. It is possible that if this approach were used for more complex applications it could save time and reduce chances for error, but it is equally possible that the time required to develop algorithms to automate these more complex calculations would offset the benefits provided. This challenge was not explored in this initial study.

\section{CONCLUSION}

This work aimed to determine if commercially available BIM tools could streamline tasks required for prefabrication. This was explored for a countertop penetration fabrication context. Through a simple, proof of concept application of BIM, it was demonstrated that the values extracted through Revit, manipulated through Dynamo, and viewed through Excel did indeed match those that would have been calculated manually. While the application chosen was very simple, the specific calculations automated would occur 
very frequently during countertop fabrication. This suggests that there is potential for this type of application to streamline the prefabrication process.

Future work will be able to explore the extent to which these benefits are observable on more complex prefabrication applications. Additionally, while the authors used a specific brand of BIM tools to generate this proof of concept, it is likely that many other BIM tools could function similarly. Therefore, the contribution of this paper is not in providing a single set of software packages for automating calculations from a model, but rather in providing a structured approach for assessing current prefabrication tasks to determine how the task might benefit from BIM-based automation.

This approach may offer value beyond countertop openings to locating other surface penetrations such as mechanical, electrical, and plumbing openings as well as skylights, windows, and doors. The value presented is a methodology with which the construction industry can extract information from a 3-D model and manipulate this information to facility producing prefabrication directives. In this simple proof of concept, besides the value of time saving, additional value is in reducing human errors, and the accuracy and precision of results as design variables change. Using this methodology to enforce constructability control limits on design may be another area for future research.

\section{ACKNOWLEDGEMENTS}

The authors of this work would like to thank the industry members who shared their current fabrication processes and discussed opportunities for improvement.

\section{REFERENCES}

Allphin (2014) BIM's past, present, and future. Available at: http://www.thefabricator.com/article/cadcamsoftware/bim-s-past-present-and-future [Accessed: 19 April 2016]

ERNSTROM (2006) AGC Guide to BIM . Available at: http://www.tpm.com/wpcontent/uploads/2013/02/AGC_Guide_to_BIM.pdf [Accessed: 19 April 2016]

Goodman (2009) 8 Steps for Better Management of Green Design and Construction Risks. Available at: https://www.greenbiz.com/blog/2009/12/10/8-steps-bettermanagement-green-design-and-construction-risks [Accessed: 19 April 2016]

Henderson (2015) BendWorksTM: Driving Efficiency in BIM and Prefabrication. Available at: https://enewsletters.constructionexec.com/techtrends/2015/09/bendworks-drivingefficiency-in-bim-and-prefabrication/ [Accessed: 19 April 2016]

Williams (2008) Implementing BIM to Drive Fabrication. Journal of Building Information Modeling (JBIM).

Yoders (2014) Prefabrication: Using BIM Technology to Remove Waste. Available at: https://lineshapespace.com/using-bim-technology-for-prefabrication-to-removewaste-and-save-money/ [Accessed: 19 April 2016] 